\title{
Massive sterile neutrinos as warm Dark Matter
}

\author{
A.D. Dolgov [1], S.H. Hansen[ \\ INFN section of Ferrara \\ Via del Paradiso 12, 44100 Ferrara, Italy
}

\begin{abstract}
We show that massive sterile neutrinos mixed with the ordinary ones may be produced in the early universe in the right amount to be natural warm dark matter particles. Their mass should be below $40 \mathrm{keV}$ and the corresponding mixing angles $\sin ^{2} 2 \theta>10^{-11}$ for mixing with $\nu_{\mu}$ or $\nu_{\tau}$, while mixing with $\nu_{e}$ is slightly stronger bounded with mass less than $30 \mathrm{keV}$.
\end{abstract}

\section{Introduction}

There seems to be convincing experimental evidence for non-zero neutrino masses and mixing angles (for a review see e.g. [1]), and if all the present day data are correct, there must exist at least one sterile neutrino species. These neutrinos should be very light (sub eV range) and hence contribute negligibly to the cosmological energy density [2]

$$
\Omega_{\nu} h^{2}=\frac{m_{\nu}}{92 \mathrm{eV}}
$$

if they were produced with the equilibrium number density in the early universe at a temperature below $\sim 10 \mathrm{MeV}$. If their number density was smaller than the equilibrium one, then the permitted value of the mass could be correspondingly higher.

One could easily envisage more than one sterile neutrino species. The masses and mixing angles of these extra neutrinos are essentially free parameters. If we consider sterile neutrinos with masses $10-200 \mathrm{MeV}$, then big bang nucleosynthesis and energy

\footnotetext{
${ }^{1}$ Also: ITEP, Bol. Cheremushkinskaya 25, Moscow 113259, Russia.

e-mail: dolgov@fe.infn.it

${ }^{3}$ e-mail: sthansen@fe.infn.it
} 
loss arguments for SN 1987A allow one to exclude mixing angles in the range $\sin ^{2} 2 \theta=$ $10^{-1}-10^{-12}$ [3], where both the upper and lower limits vary as functions of mass. On the other hand, direct terrestrial experiments exclude supplementary mixing angles in the range $\sin ^{2} 2 \theta=0.001-1$ [ [⿴囗十), and the lower limit is weaker for small masses, $m \sim 10$ $\mathrm{MeV}$. We should mention that the excluded regions only overlap for some masses, and there is still some non-excluded parameter space for $m<40 \mathrm{MeV}$ and $\sin ^{2} 2 \theta=0.01-1$.

The hypothesis that sterile neutrinos could make a considerable contribution to cosmological dark matter has a rather long history. The idea that right-handed sterile neutrinos may form warm dark matter was briefly discussed in ref. [5 and was further pursued in the paper [6]. In more detail warm dark matter cosmology was considered in ref. [7]. There are some other warm dark matter candidates discussed in the papers [8, 9]. Sterile neutrinos coming from a mirror world may also make WDM in our universe; they were discussed in the papers [10]. More models and references can be found in the recent works [11, 12]. A dark matter model with sterile neutrinos but with a non-thermal spectrum was considered in ref. [13]. Such neutrinos could be produced by the resonance oscillations in the early universe in the presence of a large lepton asymmetry. This model was further considered in ref. [14], where constraints originating from consideration of decays of $\nu_{s}$, especially of the radiative one, were presented. A detailed analysis of the essential physics of this model, namely the importance of the mixing angle suppression in the early universe, is made in ref. 15.

In this paper we find the allowed values of mass and mixing angle of a sterile neutrino, $\nu_{s}$, so that the latter could be a dominant dark matter particle. We will consider the production of $\nu_{s}$ in the early universe, calculate their energy spectrum, discuss their different decay modes, and derive bounds on mass and mixing angle from cosmology and astrophysics. 


\section{Production of $\nu_{s}$ in the early universe}

Let us consider for simplicity a two-neutrino mixing scheme, where one of the active neutrinos, $\nu_{a}=\nu_{e}, \nu_{\mu}$ or $\nu_{\tau}$, mixes with a heavy mainly sterile neutrino, $\nu_{s}$,

$$
\begin{aligned}
& \nu_{a}=\cos \theta \nu_{1}+\sin \theta \nu_{2}, \\
& \nu_{s}=-\sin \theta \nu_{1}+\cos \theta \nu_{2},
\end{aligned}
$$

where $\nu_{1}$ and $\nu_{2}$ are assumed to be the light and heavy mass eigenstates respectively, and $\theta$ is the vacuum mixing angle. We will consider small mixing angles, and hence sometimes refer to the light neutrino mass eigenstate as the active neutrino and the heavy one as sterile neutrino.

We assume that sterile neutrinos were initially absent in the primeval plasma and were produced through the mixing with active ones. As will be clear shortly, the temperature suppression of the effective mixing angle changes the production rate at high temperatures from $T^{3}$ to $T^{-9}$, so with small mixing angles the sterile neutrinos would never have reached equilibrium. As was estimated in ref. [16] the maximum ratio of the production rate to the expansion rate takes place at rather low temperatures, $T \approx 0.1 \mathrm{GeV}(\mathrm{m} / \mathrm{MeV})^{1 / 3}$. The production rate is usually approximated as [16, 17]

$$
\Gamma / H=\frac{\sin ^{2} 2 \theta_{M}}{2}\left(\frac{T}{T_{W}}\right)^{3},
$$

where $\mathrm{H}$ is the Hubble expansion parameter, $T$ is the plasma temperature, and $T_{W}$ is the decoupling temperature of the active neutrinos, which is approximately taken about $T_{W}=3 \mathrm{MeV}$. Instead of this approximate equation, below we will write down and solve the exact momentum dependent Boltzmann equation, taking into account the processes of production of $\nu_{s}$ but neglecting inverse reactions. The latter are not important if the number density of $\nu_{s}$ is small. In this more precise approach the question of the value of $T_{W}$ never appears, it is solved automatically. Another advantage of our approach here is that it permits to calculate the energy spectrum of $\nu_{s}$, while the previous method 
permitted only to estimate the total number density. Before doing these calculations it may be instructive to make the standard simplified estimates and later compare them with the exact results found below.

The mixing angle $\sin ^{2} 2 \theta_{M}$ is suppressed at large temperatures due to matter effects, and for $\nu_{\mu}$ or $\nu_{\tau}$ mixing it can be written as [18]

$$
\sin 2 \theta_{\mathrm{M}} \approx \frac{\sin 2 \theta}{1+0.8 \times 10^{-19}\left(T_{\gamma} / \mathrm{MeV}\right)^{6}\left(\delta m^{2} / \mathrm{MeV}^{2}\right)^{-1}}
$$

where the coefficient in front of the second term in the denominator was obtained by a rather arbitrary procedure of thermal averaging of the factor $\left\langle E^{2}\right\rangle \approx 12 T^{2}$, entering the ratio of the neutrino refraction index to the vacuum term $\delta m^{2} / 2 E^{2}$. We see from this expression that matter effects become essential and suppress the mixing for $T_{\gamma}>$ $0.15 \mathrm{GeV}(\mathrm{m} / \mathrm{keV})^{1 / 3}$ (a similar argument was made in ref. [6] for the left-right neutrino mixing). For the $\left(\nu_{e}-\nu_{s}\right)$-mixing the factor in the denominator should be 2.7 instead of 0.8. We will assume here that $\delta m^{2}=m_{2}^{2}-m_{1}^{2}$ is positive (specifically we assume $m_{1} \ll m_{2}$ ), and if instead the active neutrino is heavy the analysis somewhat changes (see refs. [13, 15]).

For the energy dependent calculations we need the expression for the matter effects in the denominator of eq. (4) prior to averaging over the thermal bath. The latter can be read off from the relevant equations of refs. [16, 18]

$$
\sin 2 \theta_{M}=\frac{\sin 2 \theta}{1+3.73 \cdot 10^{-20} c_{2} m(\mathrm{MeV})^{-2}\left(y^{2} / x^{6}\right)},
$$

where the $\nu_{s}$ mass, $m$, is measured in $\mathrm{MeV}$ and we used the expansion parameter of the universe, $a$, to introduce the new variables, $x=1 \mathrm{MeV} \times a, y=E a$, and neglected a possible entropy release so that the temperature drops according to $T=1 / a$. The numerical coefficient $c_{2}$ depends upon the neutrino flavour: $c_{2}=0.61$ for $\nu_{e}$ and $c_{2}=0.17$ for $\nu_{\tau}$ and $\nu_{\mu}$. However, for the temperatures close to or above the muon mass $c_{2}$ becomes the same for $\nu_{e}$ and $\nu_{\mu}$. 
The Boltzmann equation describing the evolution of the sterile neutrino distribution function, $f_{s}$, in terms of these new variables takes the form

$$
x H \partial_{x} f_{s}=I_{\text {coll }},
$$

where the collision integral is given by

$$
I_{\text {coll }}=\frac{1}{2 E_{s}} \int \frac{d^{3} p_{2}}{(2 \pi)^{3} 2 E_{2}}|A|^{2} f_{3} f_{4} d \tau_{3,4}
$$

where $d \tau_{3,4}$ is the phase space element (together with the energy-momentum $\delta$-function) of the particles $l_{3}$ and $l_{4}$ in whose collision $\nu_{s}$ is produced,

$$
l_{3}+l_{4} \rightarrow \nu_{s}+l_{2}
$$

and $f_{3,4}$ are their distribution functions. We assume that the latter are equal to their equilibrium values and then the conservation of energy gives $f_{3} f_{4}=\exp \left(-y_{1}-y_{2}\right)$ in the Boltzmann approximation. Integrating the probabilities of all the relevant processes over phase space (see the appendix) allows to find the collision integral and to solve equation (6) analytically, giving

$$
f_{s}=3.6 \cdot 10^{8} \sin ^{2} \theta\left(1+g_{L}^{2}+g_{R}^{2}\right) c_{2}^{-1 / 2} m(\mathrm{MeV})\left(\frac{10.75}{g_{*}}\right)^{1 / 2} f_{a},
$$

where $f_{a}$ is the distribution function of any of the active neutrinos, and $g_{*}$ is the number of relativistic degrees of freedom at the time when the sterile neutrinos were produced. Subsequent to the production there will be a dilution of the active neutrinos relative to the sterile ones. This is described by another factor $\left(g_{*} / 10.75\right)$.

The coefficient relating $f_{s}$ to $f_{a}$ in eq. (9) is independent on the energy of neutrinos, so the spectrum of $\nu_{s}$ remains the same as that of active neutrinos. This is somewhat surprising because the reaction rate is proportional to the neutrino energy. However, for smaller $E$ the rate becomes efficient at higher temperature, as one can see from the expression (5) describing the suppression of neutrino mixing in matter. This effect 
compensates the factor of momentum, $y$, in the kinetic equation. However, at very large temperatures, $T \sim M_{W, Z} \sim 100 \mathrm{GeV}$, the weak reaction rate drops down, and hence the spectrum will be somewhat distorted at very small $y$ 's.

It is interesting to compare the accurate results presented above with the simplified calculations based on the solution of the following approximate kinetic equation

$$
H x \partial_{x} f_{s}=\frac{1}{2} \sin ^{2} 2 \theta_{M} \Gamma_{W} f_{a},
$$

where the mixing angle and interaction rate can be taken from eqs. (3, (1). This equation is easily integrated, and we find that the result for the total number density of $\nu_{s}$ agrees within a factor of 2 with the more accurate result (9).

Up to now we have seen how the produced amount of sterile neutrinos depends on the mass and mixing angle, so let us instead ask: how many sterile neutrinos should be produced in order for them to be a dark matter candidate? Let us take $\Omega_{\mathrm{DM}}=0.3$, which means that we must demand $\rho_{s}=3 h^{2} \mathrm{keV} / \mathrm{cm}^{3}$. Using $h=0.65$ and $n_{\alpha}^{\text {today }}=100 / \mathrm{cm}^{3}$ one finds

$$
n_{s}=1.27 \times 10^{-5} n_{\alpha}\left(\frac{\mathrm{MeV}}{m}\right)\left(\frac{\Omega_{D M}}{0.3}\right)\left(\frac{h}{0.65}\right)^{2},
$$

and comparing eqs. (9) and (11) one obtains

$$
\sin ^{2} \theta=3.6 \times 10^{-14} \frac{c_{2}^{1 / 2}}{\left(1+g_{L}^{2}+g_{R}^{2}\right)}\left(\frac{10.75}{g_{*}}\right)^{1 / 2}\left(\frac{\mathrm{MeV}}{m}\right)^{2} .
$$

This equation thus describes a line in mass-mixing parameter space, where the sterile neutrino must lie, if it indeed is the dominant dark matter particle. Let us now see how decay processes and supernovae can further restrict this parameter space.

\section{Decay}

The mixing couples the heavier $\nu_{2}$ to the Z-boson, and allows for the decay channel

$$
\nu_{2} \rightarrow \nu_{1}+\ell+\bar{\ell}
$$


where $\nu_{1}$ is mostly an active flavour and $\ell$ is any lepton with a mass smaller than half the mass of the heavy neutrino. This mixing angle can be translated into decay time

$$
\tau=\frac{10^{5} f(m)}{m(\mathrm{MeV})^{5} \sin ^{2} 2 \theta} \sec ,
$$

where $f(m)$ takes into account the open decay channels (for $m<1 \mathrm{MeV}$ only the neutrino channels are open, and $f(m)=0.86$, while for $m_{s}>2 m_{e}$ the $e^{+} e^{-}$-channel is also open and $f(m)=1)$. Now, for the sterile neutrino to be a dark matter candidate we must demand that it does not decay on cosmic time scales, which means $\tau>4 \times 10^{17}$ sec, and hence from eq. (14) we get

$$
\sin ^{2} 2 \theta<2.5 \times 10^{-13} \frac{f(m)}{m(\mathrm{MeV})^{5}} .
$$

We can, however, get an even stronger bound by considering the radiative decay

$$
\nu_{s} \rightarrow \nu_{a}+\gamma
$$

where $\nu_{a}$ is any of the active neutrinos. This decay will contribute with a distinct line into the diffuse photon background near $m / 2$. The branching ratio for the reaction (16) was found 19 to be: $B R \approx 1 / 128$. The flux of electromagnetic radiation from the decay was calculated in the papers [20, 21] (see also refs. [14, 22]). In the case of a large life-time, larger than the universe age, and of the matter dominated flat universe the intensity of the radiation in the frequency interval $d \omega$ is equal to

$$
d I=(B R) \frac{n_{s}^{(0)}}{H \tau_{s}} \frac{\omega^{1 / 2} d \omega}{\left(m_{s} / 2\right)^{3 / 2}}
$$

where $n_{s}^{(0)}$ is the present day number density of $\nu_{s}$ and $H$ is the Hubble constant. Here we neglected corrections related to a possible dominance of the lambda-term in the latest history of the universe.

In the energy range interesting to us a rather conservative upper limit for the flux can be read of the figure of ref. 23]

$$
\frac{d \mathcal{F}}{d \Omega}<0.1\left(\frac{1 \mathrm{MeV}}{E}\right) \mathrm{cm}^{-2} \mathrm{sr}^{-1} \mathrm{sec}^{-1}
$$


and taking the values $\Omega_{s}=0.3$ and $h=0.65$ we find: $\tau>4 \times 10^{22}$, which together with eq. (14) leads to the bound

$$
\sin ^{2} 2 \theta<2.5 \times 10^{-18} \frac{f(m)}{m(\mathrm{MeV})^{5}} .
$$

A mass dependent bound can be found by considering the energy loss argument for $\mathrm{SN} 1987 \mathrm{~A}$, for $m_{s}<3 T_{S N} \approx 100 \mathrm{MeV}$. Sterile neutrinos produced due to mixing with the active ones inside the supernova would carry away too much energy, hence shortening the explosion. The excluded mixing angles have been calculated several times for SN 1987A, and the results are about $\sin ^{2} 2 \theta<3 \times 10^{-8}$ for $\nu_{\mu}$ or $\nu_{\tau}$ mixing [3] and $\sin ^{2} 2 \theta<10^{-10}$ for the $\nu_{e}$ mixing [24] for masses of the order MeV. For smaller masses, $m<40 \mathrm{keV}$, this bound weakens substantially due to matter effects, since the matter mixing angle can be expressed as [25]

$$
\sin ^{2} 2 \theta_{M}=\frac{\sin ^{2} 2 \theta}{\sin ^{2} 2 \theta+\left(\cos 2 \theta+1.4 \cdot 10^{3}\left(\frac{\mathrm{keV}^{2}}{m^{2}}\right)\right)^{2}} .
$$

On fig. 1 one sees, that this SN-bound is weaker than the diffuse gamma background limit for all neutrino masses.

Plotting the equation describing the production, eq. (12), together with the bounds from SN 1987A and radiative decay, makes it clear that $\left(\nu_{\alpha}-\nu_{s}\right)$-mixing (see fig. 1) as the producer of dark matter demands that the neutrino mass must be smaller than $40 \mathrm{keV}$ for $\nu_{\mu}$ or $\nu_{\tau}$ mixing (solid lines), and slightly below this values for $\nu_{e}$ mixing (dashed line), and with corresponding mixing angle $\sin ^{2} \theta>10^{-11}$. The two thinner full lines in the figure allow for a factor 2 uncertainty in the amount of dark matter, $\Omega_{D M}=0.15-0.60$.

A lower bound on the dark matter particle can be obtained from large scale structure. A small mass of the dark matter particle will erase structure on small scale, and present day data seem to exclude masses smaller than a few tens of $\mathrm{eV}$.

Several comments are in order here. First we must check that the sterile neutrinos are indeed relativistic when produced. This is the case, because the temperature $T_{\max }$ is 


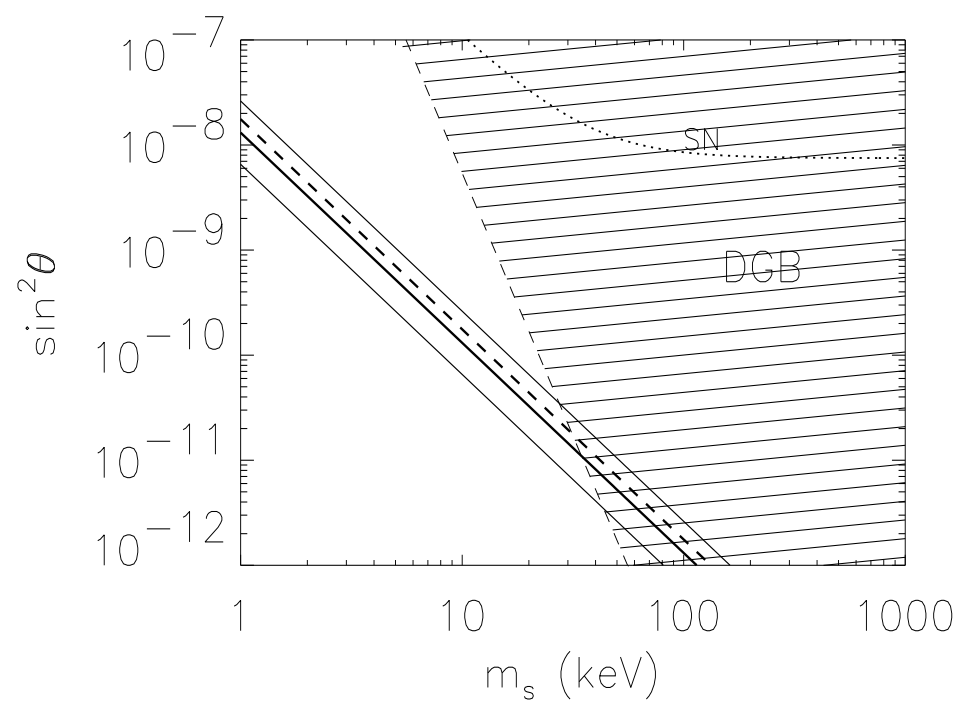

Figure 1: Bounds from $\left(\nu_{\alpha}-\nu_{s}\right)$-mixing. The middle full line describes the mass-mixing relationship if sterile neutrinos are the dark matter for $\left(\nu_{\tau}-\nu_{s}\right)$-mixing. The two other full lines allow a factor 2 uncertainty in the amount of dark matter, $\Omega_{D M}=0.15-0.6$. The dashed line is for $\left(\nu_{e}-\nu_{s}\right)$-mixing. The hatched region for big masses is excluded by the Diffuse Gamma Background. The region above the dotted line is excluded by the duration of SN 1987A for $\left(\nu_{\tau}-\nu_{s}\right)$-mixing.

about $1.3 \mathrm{GeV}$ for $m=1 \mathrm{MeV}$, and about $0.13 \mathrm{GeV}$ for $m=1 \mathrm{keV}$. Further, the dilution factor is somewhere between 1 and 4 depending upon whether the production happens before or after the QCD transition, and can thus enlarge the allowed region slightly compared to the figures, where we for simplicity used $g_{*}=10.75$. Looking at eq. (9) it seems that the sterile neutrinos follow an equilibrium distribution function. This is not quite the case, because the small momentum neutrinos are produced first, and hence their relative importance is increased by the subsequent entropy release (which dilutes the active neutrinos). A different non-thermal effect can appear for $\left(\nu_{\mu}-\nu_{s}\right)$-mixing, since the factor $c_{2}$ is 0.17 when the $\mu$ 's are absent (for $T \ll m_{\mu}$ ), whereas it grows to $c_{2}=0.61$ when the muons are fully present in the plasma. This means that bigger momenta will be produced with a factor 1.9 more efficiently [26]. 


\section{Discussion and conclusion}

The model considered in this paper, is undoubtly the simplest, oldest and, as we have seen, very natural for warm dark matter. The value of the $\nu_{s}$ mass can in the future be found from the detailed analysis of large scale structure formation. As we have seen this model permits masses about $\mathrm{keV}$ which may be interesting for the galaxy formation problem [11, and furthermore recent N-body simulations of large scale structure compared with observations of the number of satellite galaxies have in fact indicated that the dark matter particle may have a mass about keV [27. It is interesting to note, that a keV sterile neutrino has been suggested as a possible explanation of the observed pulsar velocities [28]. Finally, better observations of the diffuse $\gamma$ background around $\mathrm{keV}$ energies should be able to cut away more of the parameter space, or potentially make an indirect observation of dark matter.

\section{Acknowledgement}

It is a pleasure to thank K. Abazajian, Z. Berezhiani and G. Fuller for interesting and fruitful conversations, in particular SH thanks G. Fuller for presenting ref. [15 prior to publication. AD is grateful to the Theory Division at CERN for hospitality while this work was done.

\section{A Solving the Boltzmann equation}

All the relevant processes were presented in table 2 of ref. [3]. There are two kinds of matrix elements, namely $\left(p_{1} p_{2}\right)\left(p_{3} p_{4}\right)$ and $\left(p_{1} p_{4}\right)\left(p_{3} p_{2}\right)$, and one finds from the integral over phase space that

$$
\int d \tau_{34}\left(p_{1} p_{2}\right)\left(p_{3} p_{4}\right)=3 \int d \tau_{34}\left(p_{1} p_{4}\right)\left(p_{3} p_{2}\right)=\frac{\left(p_{1} p_{2}\right)^{2}}{8 \pi}
$$


Now one can count all the relevant processes, integrate over momenta and find

$$
H x \partial_{x} f_{s}=\frac{5 \times 2^{4}}{3 \pi^{3}} \sin ^{2} \theta\left(1+g_{L}^{2}+g_{R}^{2}\right) G_{F}^{2} E_{1} T^{4} f_{a}
$$

where $H x=4.5 \times 10^{-22}\left(\frac{g_{*}}{10.75}\right) x^{-1} \mathrm{MeV}$ and $G_{F}=1.1664 \times 10^{-5} \mathrm{GeV}$. With the variable $\xi=y / x^{3}$ and $\beta$ defined in eq. (5) the suppression of mixing angle is

$$
\sin 2 \theta_{M}=\frac{\sin 2 \theta}{1+\beta^{2} \xi^{2}},
$$

and with the integral

$$
\int_{0}^{\infty} d \xi\left(\frac{1}{1+\beta^{2} \xi^{2}}\right)^{2}=\frac{4}{\pi} \frac{1}{\beta},
$$

we find the result in eq. (9).

\section{References}

[1] J. Ellis, Nucl. Phys. Proc. Suppl. 91 (2000) 503 hep-ph/0008334.

[2] S.S. Gerstein, Ya.B. Zeldovich, ZhETF Pis'ma Red. 4 (1966) 174;

R. Cowsik and J. McClelland, Phys. Rev. Lett. 29 (1972) 669.

[3] A. D. Dolgov, S. H. Hansen, G. Raffelt and D. V. Semikoz, Nucl. Phys. B 580 (2000) 331 hep-ph/0002223;

A. D. Dolgov, S. H. Hansen, G. Raffelt and D. V. Semikoz, Nucl. Phys. B 590 (2000) 562 hep-ph/0008138.

[4] NOMAD Collaboration, hep-ex/0101041.

[5] K.A. Olive and M.S. Turner, Phys. Rev. D25 (1982) 213.

[6] S. Dodelson and L. M. Widrow, Phys. Rev. Lett. 72 (1994) 17.

[7] S. Colombi, S. Dodelson, and L.M. Widrow, Astrophys. J. 458 (1996) 1. 
[8] R. A. Malaney, G. D. Starkman and L. Widrow, Phys. Rev. D52 (1995) 5480.

[9] G. Dvali and Y. Nir, JHEP 9810 (1998) 014.

[10] Z.G. Berezhiani and R.N. Mohapatra Phys. Rev. D52 (1995) 6607;

Z.G. Berezhiani, A.D. Dolgov, and R.N. Mohapatra, Phys.Lett. B375 (1996) 26;

Z.G. Berezhiani, Acta Phys.Polon. B27 (1996) 1503.

[11] J. Sommer-Larsen and A.D. Dolgov, [astro-ph/9912166].

[12] S. Hannestad, astro-ph/0008451.

[13] X. Shi and G. M. Fuller, Phys. Rev. Lett. 82 (1999) 2832.

[14] M. Drees and D. Wright, hep-ph/0006274.

[15] G. Fuller, "Comment on "New constraints on cool Dark Matter"”, to appear.

[16] R. Barbieri and A.D. Dolgov, Phys. Lett. B237 (1990) 440;

K. Kainulainen, Phys. Lett. B244 (1990) 191.

[17] K. Enqvist, K. Kainulainen, and M.Thomson, Nucl. Phys. B373 (1992) 498;

A.D. Dolgov, hep-ph/0006103.

[18] D. Nötzold and G. Raffelt, Nucl. Phys. B307 (1988) 924.

[19] V. Barger, R. J. Phillips and S. Sarkar, Phys. Lett. B352 (1995) 365.

[20] F.W. Stecker, Phys. Rev. Lett. 45 (1980) 1460.

[21] R. Kimble, S. Boyer, and P. Jacobsen, Phys. Rev. Lett. 46 (1981) 80.

[22] E. W. Kolb and M. S. Turner, "The Early Universe," Redwood City, USA: AddisonWesley (1990).

[23] M. T. Ressell and M. S. Turner, FERMILAB-PUB-89/214-A. 
[24] K. Kainulainen, J. Maalampi, and J.T. Peltoniemi, Nucl. Phys. 358 (1991) 435;

G. Raffelt and G. Sigl, Astropart. Phys. 1 (1993) 165.

[25] G. G. Raffelt, Chicago, USA: Univ. Pr. (1996) $664 p$.

[26] Lacking a better synonym than lukewarm dark matter, we decide to use the standard name sterile neutrinos (see [11, 13] for great names like warmons or coolDM).

[27] P. Colin, V. Avila-Reese and O. Valenzuela, Astrophys. J. 542 (2000) 622.

[28] A. Kusenko and G. Segre, Phys. Lett. B396 (1997) 197;

A. Kusenko, astro-ph/9903167. 